\title{
A Comparison of Diagnostic Performance of Signal Intensity and Volume Related MRI Multiparameters for Assessing Different Response of Rectal Cancer to Neoadjuvant Chemoradiotherapy
}

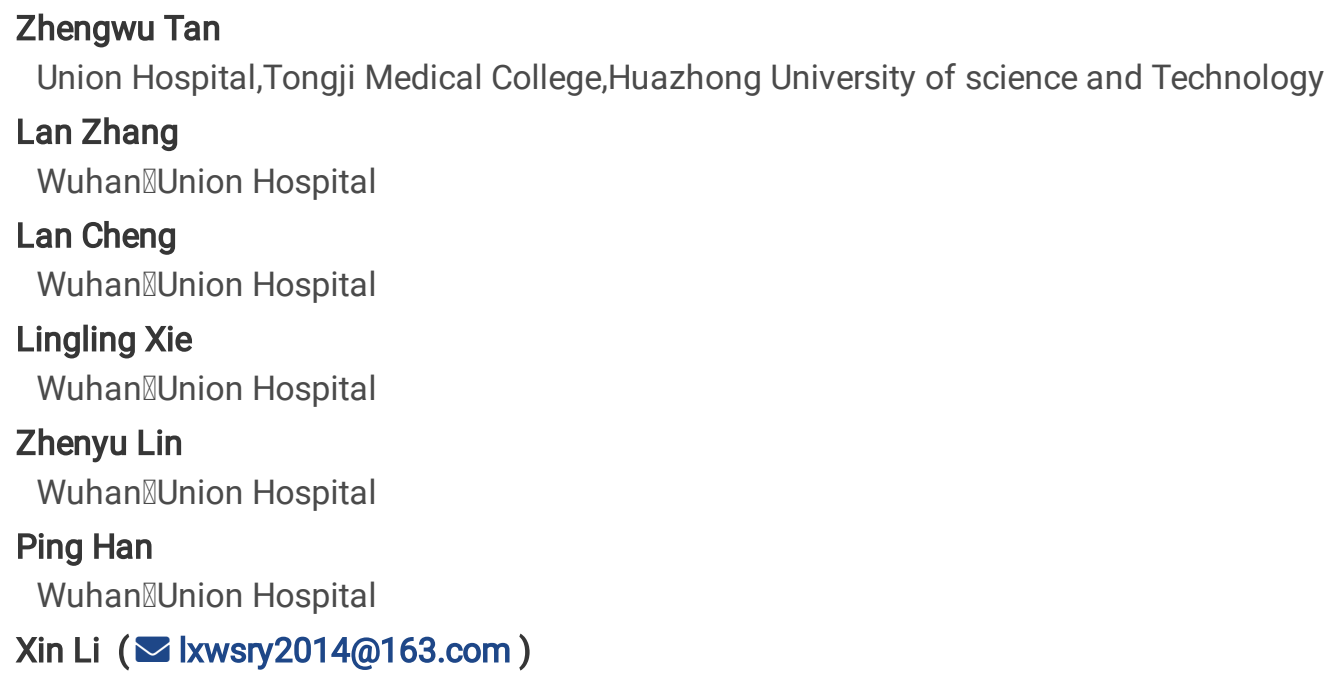




\section{Abstract}

Background: "Wait-and-see", has been proposed as a possible method of treatment in patients with locally advanced rectal cancer (LARC) after chemoradiotherapy (CRT), MR is important to predict the pathological tumor regression grade(TRG) to preoperative CRT. This study aims to evaluate the diagnostic value of signal intensity $(\mathrm{SI})$ and volume $(\mathrm{V})$ change rate in magnetic resonance imaging (MR) and determine which ones perform best as a potential biomarker for predicting pathological TRG to preoperative CRT in patients with LARC.

Methods: A retrospective analysis of 82 patients with LARC, for whom clinical and imaging data were retrieved from our institute was conducted between Oct 2017and Oct 2019. Patients underwent pre- and post-CRT T2-weighted (T2W), diffusion-weighted (DW)/apparent diffusion coefficient (ADC) and contrast-enhanced T1-weighted (ceT1W). V, difference of volume between pre-CRT and post-CRT tumor $(\triangle \mathrm{V}), \mathrm{V}$ of tumor reduction rate $(\% \triangle \mathrm{V})$, as well as SI of tumor (SIt), SI of muscle (SIm), relative SI ratio of tumor/muscle (SIR), changed difference SIR between pre- and post-CRT SIR ( $\triangle \mathrm{SIR})$, SIR of tumor changed rate (\% $\triangle \mathrm{SIR})$ on T2W, $A D C$ and ceT1W were measured. All of LARC after CRT were confirmed pathologically and classifed into histologic TRG: TRG 0 (complete response), TRG 1 (moderate response), TRG 2 (minimal response), TRG 3 (poor response). Descriptive statistics and areas under the receiver operating characteristic curves (ROC) were generated to compare performance of $\% \triangle V$ and $\% \triangle S I R$ on T2W, DW, ceT1W for distinguishing between different pathological TRG.

Result: Of the 82 patients, TRG 0 (16), TRG 1 (15), TRG 2 (35), TRG 3 (16).Except for $A D C$ - $\% \triangle S I R$, the remaining \% $\triangle \mathrm{V}$ and $\% \triangle S I R$ on T1W, ADC/DWI, ceT1W showed statistics significance between four groups. There was not distinguishable between TRG 1 and TRG 2, TRG 2 and TRG 3 by $\% \triangle V$ and/ or $\% \triangle S I R$, the remaining different TRG all were identified by $\% \triangle V$ and/ or $\% \triangle S I R$ on $T 2 W$, $A D C / D W I$, ceT1W. Compared with other individual \% $\triangle \mathrm{V}$ or $\% \triangle \mathrm{SIR}$, the combination of $D W-\% \triangle \mathrm{V}$ and $T 2 \mathrm{~W}-\% \triangle \mathrm{SIR}(\mathrm{DW}-\mathrm{C} \triangle \mathrm{V}$ * T2W-\% $\triangle$ SIR) yielded higher AUCs to predict TRG 0 from TRG 2 (AUCs: 0.954, sensitivity: $93.75 \%$, specificity: $97.14 \%$ ) and TRG 3 (AUCs: 1.000 , sensitivity: $100 \%$, specificity: $100 \%$ ), although AUC of all had not significant differences between TRG groups. there was statistically significant differences in post-CRT T restage and ypT stage between fours groups, respectively, but the agreement between post-CRT T restage and ypT is low ( kappa=0.191).

Conclusions: $V$ and/or SIR change rate on T2W, DW, ceT1W with high diagnostic performance could be useful in differentiating complete response from non-complete response; SIR change rate could be useful for distinguishing between moderate response and poor response.

\section{Background}

The multidisciplinary treatment of locally advancedrectal cancer (LARC) has markedly improved and led to better patient outcomes over the last three decades. The reasons for this are multifactorial, but one important factor is the use of Neoadjuvant chemoradiation (CRT) [1, 2]. Tumors after CRT will have different TRG and downsizing, and has been shown to be an independent and important prognosticating factor for survival [3]. Therefore, it is important to predict the tumour regression grade (TRG) before surgery because of approximately $10-30 \%$ of rectal cancer patients achieve pathological complete remission (pCR) after CRT[1, 2, 4]. A wait-and-see policy has been proposed as a possible method of treatment in clinical complete responders $[2,4]$.

MR as optional non-invasive examination has also played an increasingly important role and become the gold standard for rectal cancer staging and assessment of response to neoadjuvant treatment as restaging after CRT[4]. mrTRG as a potential biomarker for predicting PTRG to preoperative CRT and disease-free survival and overall survival in patients with LARC, was reported[1, 510]. However, mrTRG suffers from different interreader agreement. Siddiqui et al reported a wide range of $\mathrm{k}$ values $(0.14-0.82)$, and the assessment of the response of rectal cancers to chemoradiation therapy may be performed effectively using mrTRG[7]. while Hotker et al and Sclafani et al reported the agreement between mrTRG and pTRG is low $(0.10,0.24$, respectively) and mrTRG cannot be used as a surrogate of PTRG[4, 8], because it is difficult to distinguish between the signal intensity (SI) of fibrosis and viable tumors by visual inspection[3,11]. Consequently, the significance of quantitative analysesand functional imaging studies, along with morphological evaluations have been proposed to assess the TRG after CRT including SI and volume (V) on MR related pammeters respectively, and showed promising results for predicting $\mathrm{pCR}[8,11-16]$. Meanwhile studies have reported SI and $V$ showed potential limited additional diagnostic value for pathological good responders[2, 8, 13, 17].

Page 2/16 
In previous study, SI and V was used to discriminate pCR or response groups from non-pCR or non response groups, and not for discrimination between TRG groups. To our knowledge, different TRG after CRT has DFS and OS in patients withLARC[7, 9], the determination of a TRG before surgery would influence the subsequent treatment choice, so an accurate clinical assessment of response becomes essential. However, MR quantitative was evaluated to distinguish different TRG has not been reported, we hypothesized that relative SI (SIR) and V change rate on MR sequence included T2WI, DW/ADC, ceT1W between after and after CRT was also associated with TRG, and to evaluate the diagnostic value of their and determine which ones perform best as a potential biomarker for predicting PTRG to preoperative CRT in patients with LARC.

\section{Materials And Methods}

This retrospective study was approved by the relevant institutional review board and the need to obtain informed consent was waived. Clinical data were obtained consecutively from the Hospital Database between october 2017 and october 2019.

\section{Patiens}

The inclusion criteria of patients were: (1) histologically (biopsy-) confrmed rectal adenocarcinoma; (2) locally advanced disease (staged on pre-CRT MR images as cT3-4 and/or N-category positive); (3) completed standard CRT, followed by total mesorectal excision (TME); (4) availability of pre- and post-CRT MR imaging results,including T2-weighted(T2W), diffusion-weighted (DW)/apparent diffusioncoefficient (ADC), non contrast-enhanced T1-weighted (nonceT1W) and contrast-enhanced T1-weighted (ceT1W) imaging results; (5) standard TME within 8-10 weeks of completing the full CRT course[18].Exclusion criteria were: (a) nonresectable and/or metastatic disease; (b) insufficient MR image quality; (c) not complete CRT or underwent TME; (d) tumor with signet ring cell carcinoma after TME, with several high-signal mucus components by using T2WI and numerous postoperative pathological mucus lakes (greater than 50\%), (e) patients with an interval > 10 weeks between post-CRT MRI and surgery, because a large interval between the post-CRT MRI and the surgery can cause the possibility of tissue changes during the delayed period after MRI[2](Fig. 1).

\section{Magnetic resonance imaging protocol}

All patients were performed atmultiple MR unit included 1.5 and 3.0 T by using a phasedarray body coil, Without any bowel preparation. Oblique axial or axial T2-weighted (T2W), contrast-enhanced T1- weighted (ceT1W) images and ADC images were retrieved from the picture archiving and communication system (PACS, Carestream, Canada). Routine rectal MR protocol and image acquisition parameters are presented in Appendix A1. The first MRI examination was performed to assess tumor stage, the preoperative MRI to assess treatment response and restage after CRT.

\section{Volumetric Image Evaluation}

One gastrointestinal radiologists with respective 7 years of expertise in rectal cancer diagnosis, calculated tumor V and SI by manually tracing the tumor boundaries on the axial images and placing free-hand regions-of-interest (ROIs), which provided the sectional area of the lesion for each tumor-containing section (Fig. 2). On the T2W images, tumor was defined as areas of isointense or hyperintense signal as compared with the relatively lower hypointense signal of the normal adjacent muscular rectal wall. On the DW images, areas of high SI, compared with the normal bowel wall or background of lower SI tissue, were considered as tumor, portions of the tumor showing a high DWI signal along with a high ADC were avoided, so as not to include T2 shinethrough in our ADC data; On the ceT1W images, Areas of enhanced high SI, compared with the normal bowel wall, were considered as enhanced tumor. On post-CRT T2-weighted MR images, areas of markedly low SI at the location of the primary tumor bed were interpreted as fibrosis. As the risk for residual tumor in these fibrotic areas is known to be $\pm 50 \%$, they were also included in the volumetric and SI measurements[13].

Whole-tumor volume was then calculated by multiplying each crosssectional area by section thickness (Fig. 2V1-V4). Post-CRT measurements were performed with comparison to pre-CRT MR images to ensure ROls were placed within same axial level of the location of the primary tumor, The ROls with maximum area of tumor obtained on single sections of axial T2W, ADC, nonceT1W, ceTW. The intestinal lumen and the artifact areas were avoided after ROI selection. In some patients, high signal-intensity zones

Page 3/16 
were not identifed on post-CRT DW images, and then the ROIs were positioned at the location of the tumor bed before CRT (Fig. 3). The SI of iliopsoas muscle (SIm) was used as reference tissue carefully avoiding any intramuscular fat[8, 19-21].

The $\Delta$ was defined as the change in $V$ and SI value between pre-CRT and post-CRT measurements, while $\% \Delta$ was defined as the change rate. $\mathrm{V}$ and $\mathrm{SI}$ was calculated using the following formula:

$\mathrm{T} 2 \mathrm{~W}-\% \triangle \mathrm{V}=(\mathrm{T} 2 \mathrm{~W}-\mathrm{V}$ pre $-\mathrm{T} 2 \mathrm{~W}-\mathrm{Vpost}) / \mathrm{T} 2 \mathrm{~W}-\mathrm{Vpre} \times 100$

DW-\% $\triangle \mathrm{V}=(\mathrm{DW}-\mathrm{Vpre}-\mathrm{DW}-\mathrm{Vpost}) / \mathrm{DW}-\mathrm{V} p r e \times 100$

ceT1W-\% $\triangle \mathrm{V}=($ ceT1W-Vpre - ceT1W-Vpost $) /$ ceT1W pre $\times 100$

SIR $=$ SIt $/$ SIm (SI of the tumour/ SI of the iliopsoas muscle[8].)

$\% \triangle S I R=($ SIRpre - SIRpost $) /$ SIRpre

T2W-\% $\triangle$ SIR $=($ T2W-SIRpre - T2W-SIRpost $) /$ T2W-SIRpre $\times 100$

DW-\% $\triangle$ SIR $=($ DW-SIRpre - DW-SIRpost $) /$ DW-SIRpre $\times 100$

ceT1W- $\triangle$ SIRpre = ceT1W-SIRpre(contrastenhanced-T1W) - nonceT1W-SIRpre(non contrastenhanced-T1W)

ceT1W- $\triangle$ SIRpost $=$ ceT1W-SIRpost - nonceT1W-SIRpost

ceT1W-\% $\triangle$ SIR=(ceT1W- $\triangle$ SIRpre - ceT1W- $\triangle$ SIRpost)/ceT1W- $\triangle$ SIRpre $\times 100$

Another radiologist with 10 years of expertise in gastrointestinal diagnostics independently assessed each pre and post-treatment MRI, blinded to clinical and histopathological information, patients and MRI image characteristics that were evaluated (Table 1) 
Table 1

Clinical characteristics and MRI characteristics in TRG groups patients

\begin{tabular}{|c|c|c|c|c|c|c|}
\hline Characteristics & & $\begin{array}{l}\text { TRG } 0 \\
(16)\end{array}$ & $\begin{array}{l}\text { TRG } 1 \\
\text { (15) }\end{array}$ & $\begin{array}{l}\text { TRG } 2 \\
\text { (35) }\end{array}$ & $\begin{array}{l}\text { TRG } 3 \\
(16)\end{array}$ & $\mathbf{P}$ \\
\hline Gender & Man & 12 & 12 & 24 & 13 & 0.737 \\
\hline Age & & $\begin{array}{l}48.94 \pm \\
10.22\end{array}$ & $\begin{array}{l}53.20 \pm \\
8.14\end{array}$ & $\begin{array}{l}52.83 \pm \\
10.04\end{array}$ & $\begin{array}{l}50.44 \pm \\
12.17\end{array}$ & 0.542 \\
\hline \multirow[t]{8}{*}{ MR unit } & Pre- /post-CRT & & & & & $0.705 / 0.339$ \\
\hline & Siemens Verio & $1 / 0$ & $1 / 0$ & $2 / 6$ & $4 / 1$ & \\
\hline & siemens TrioTim & $2 / 2$ & $0 / 2$ & $4 / 1$ & $3 / 1$ & \\
\hline & Siemens skyra & $2 / 1$ & $1 / 2$ & $1 / 3$ & $1 / 1$ & \\
\hline & Siemens Avanto & $6 / 7$ & $7 / 6$ & $17 / 18$ & $6 / 10$ & \\
\hline & Siemens Aera & $5 / 6$ & $5 / 3$ & $9 / 7$ & $2 / 2$ & \\
\hline & Philips Ingenia & $0 / 0$ & $1 / 1$ & $1 / 0$ & $0 / 1$ & \\
\hline & GE 750w & $0 / 0$ & $0 / 1$ & $1 / 0$ & $0 / 0$ & \\
\hline \multirow[t]{4}{*}{ Tumor differentiation } & & & & & & 0.532 \\
\hline & Well differentiated & 0 & 1 & 4 & 1 & \\
\hline & $\begin{array}{l}\text { Moderately } \\
\text { differentiated }\end{array}$ & 15 & 13 & 30 & 14 & \\
\hline & $\begin{array}{l}\text { Poorly } \\
\text { differentiated }\end{array}$ & 1 & 1 & 1 & 1 & \\
\hline \multirow[t]{3}{*}{ Pre-nCRT T stage (n) } & & & & & & 0.278 \\
\hline & T4 & 3 & 7 & 16 & 7 & \\
\hline & T3 & 13 & 8 & 19 & 9 & \\
\hline \multirow[t]{5}{*}{ Post-CRT MR restage (n) } & & & & & & 0.000 \\
\hline & $\mathrm{T} 4$ & 0 & 5 & 5 & 4 & \\
\hline & T3 & 4 & 8 & 20 & 11 & \\
\hline & $\mathrm{T} 2$ & 8 & 2 & 10 & 1 & \\
\hline & T0 & 4 & 0 & 0 & 0 & \\
\hline $\begin{array}{l}\text { Interval between pre- and post- } \\
\text { CRMR (d) }\end{array}$ & & $\begin{array}{l}91.44 \pm \\
19.29\end{array}$ & $\begin{array}{l}118.87 \pm \\
96.29\end{array}$ & $\begin{array}{l}111.49 \pm \\
74.72\end{array}$ & $85 \pm 39.1$ & 0.356 \\
\hline $\begin{array}{l}\text { interval between post-CRT MR } \\
\text { and surgery (d) }\end{array}$ & & $\begin{array}{l}17.25 \pm \\
10.75\end{array}$ & $\begin{array}{l}16.87 \pm \\
12.84\end{array}$ & $\begin{array}{l}16.86 \pm \\
12.86\end{array}$ & $\begin{array}{l}9.63 \pm \\
7.58\end{array}$ & 0.156 \\
\hline \multirow[t]{6}{*}{ ypT stage } & & & & & & 0.000 \\
\hline & T4 & 0 & 2 & 3 & 4 & \\
\hline & T3 & 0 & 5 & 16 & 8 & \\
\hline & $\mathrm{T} 2$ & 0 & 7 & 14 & 4 & \\
\hline & T1 & 0 & 1 & 2 & 0 & \\
\hline & TO & 16 & 0 & 0 & 0 & \\
\hline
\end{tabular}

\section{Pathological evaluation after CRT}


Surgically resected specimens were pathologically analyzed according to the seventh edition of the American Joint Committee onCancer (AJCC) TNM staging system and according Ryan et al. staging systems[22, 23], including tumor staging (T0 - T4b) and pathologic tumor regression grade (TRG) was collected. Patients were categorized based on their response to therapy, assessed by TRG, into the following 4 groups: (1) TRG 0: complete response with no viable cancer cells, (2) TRG 1: moderate response with single cancer cells or small groups of cancer cells, (3) TRG 2: minimal response with residual cancer outgrown by fibrosis, and (4) TRG 3: poor response with minimal or no tumor kill, extensive residual cancer.

\section{Statistical analysis}

Data were expressed as numbers and percentages for categorical variables and as means \pm standard deviations for continuous variables. The Kruskal Wallis test and $\chi 2$ test was used to assess the relationship between continuous variables and categorical variables, respectively. The thresholds of $\% \Delta$ values to predict tumor responsiveness TRG were assessed by using receiver operating characteristic (ROC) curve analysis, according to the nearest point to the upper left corner in the diagram of ROC curves, as well as their combinations were assessed with logistic regression analysis using Firth bias-correction[17]; Differences in diagnostic performance were analyzed by comparing the ROC curves according to the method described by DeLong et al[24]. The $\mathrm{K}$ test was used to assess agreement between post-CRT restanging MR and pathological ypT stage. Statistical analysis was performed using the SPSS 19.0, medcalc 19.0.4 and GraphPad Prism 7.0 software. P-values were calculated using two-sided tests, and p-values $<0.05$ were considered significant.

\section{Results}

A total of 82 patients with LARC, with an average age of 51.67 years \pm 10.16 (range, 27-71 years), were enrolled in this study based on the inclusion and exclusion criteria in the materials and methods section, including 61 men (74.39\%) and 21 women (25.61\%). Among of 84 patients, TRG 0 was 16 (19.51\%), TRG 1 was 15 (18.29\%), TRG 2 was 35 (42.68\%), TRG 3 was 16 (19.51\%).

\section{Patient and Treatment Characteristics}

There was no statistical difference in gender, age, MR unit, tumor differentiation, pre-CRT T stage, interval between pre- and postCRT MR, interval between post-CRT restaging MR and surgery between TRG groups (Table 1); The median time between pre- and post-CRT MR, and the restaging MRI and surgery was 92.5 days (range, 31-498 days) and 12 days (range, 1-63 days),

respectively. All of patients were staged on MR images pre-CRT as cT3-4 (cT3: $59.77 \%$ vs cT4: $40.23 \%$ ). On the other hand, There was statistical difference in post-CRT MR T restaging and pathological ypT stage between TRG groups (both P<0.001), respectively, agreement of which was low (Kappa $=0.191$ ); post-CRT T restage show $43.9 \%$ of all were downstaged, but in fact, $70.73 \%$ of all were downstaged on pathology (Table 2 ).

Table 2

Agreement of post-CRT clinical T restage and ypT stage

\begin{tabular}{|llllll|}
\hline \multirow{2}{*}{ post-CRT T restage } & \multicolumn{4}{l}{ ypT stage } \\
\cline { 2 - 6 } & Yp T0 & Yp T1 & Yp T2 & Yp T3 & Yp T4 \\
\hline CT 0 & 4 & 0 & 0 & 0 & 0 \\
\hline CT 1 & 8 & 2 & 4 & 7 & 0 \\
\hline CT 2 & 4 & 1 & 18 & 19 & 1 \\
\hline CT 3 & 0 & 0 & 3 & 3 & 8 \\
\hline CT 4 & 16 & 3 & 25 & 29 & 9 \\
\hline
\end{tabular}

\section{T2W, DW, ceT1W MR SI value and Volumetry}

There was no statistical difference in Vpre, SImpre, SItpre, SIRpre, SImposton T2W, DW, ceT1W and nonceT1W between groups, as well as SI, SIR, $\triangle S I R$ on ADC between groups. There was statistical difference in nonceT1W-SItpost $(p=0.035)$, but no statistical 


\section{3. \% $\triangle$ SIR and $\% \triangle V$ on T2W, DW and ceT1W MR between four groups}

Except for $A D C-\% \triangle S I R$, the remaining $\% \triangle V$ and $\% \triangle S I R$ on $T 1 W, A D C / D W I$, ceT1W showed statistics significance between four groups (Table 3); $T 2 W-\% \triangle V, D W-\% \triangle V$, ceT1W-\% $\triangle V, T 2 W-\% \triangle S I R$, ceT1W-\% $\triangle$ SIR were reduced with grading of respons after CRT that from 0 to 3 .

Table 3

Comparison of $\% \triangle V$ and $\% \triangle S I R$ on $T 1 W, A D C / D W I$, ceT1W between fours groups.

\begin{tabular}{|llllll|}
\hline Parameter & TRG 0 (\%) & TRG 1 (\%) & TRG 2 (\%) & TRG 3 (\%) & P \\
\hline T2W-\% $\triangle \mathrm{V}$ & $86.01 \pm 15.33$ & $43.73 \pm 31.31$ & $37.48 \pm 31.53$ & $23.27 \pm 27.79$ & 0.000 \\
\hline DW-\% $\triangle \mathrm{V}$ & $88.89 \pm 12.95$ & $54.62 \pm 31.13$ & $42.54 \pm 24.63$ & $32.63 \pm 29.7$ & 0.000 \\
\hline ceT1W\% $\triangle \mathrm{V}$ & $81.99 \pm 14.93$ & $48.81 \pm 22.37$ & $39.06 \pm 25.84$ & $22.30 \pm 28.45$ & 0.000 \\
\hline T2W-\% $\triangle \mathrm{SIR}$ & $44.73 \pm 8.95$ & $29.39 \pm 13.28$ & $6.85 \pm 38.31$ & $0.2 \pm 32.12$ & 0.000 \\
\hline ADC-\% $\triangle \mathrm{SIR}$ & $-301.19 \pm 880.12$ & $-16.24 \pm 51.95$ & $-72.94 \pm 401.05$ & $-41.9 \pm 111.42$ & 0.775 \\
\hline ceT1W-\% $\triangle \mathrm{SIR}$ & $34.02 \pm 36.18$ & $17.62 \pm 32.76$ & $-11.42 \pm 53.09$ & $-26.67 \pm 40.74$ & 0.001 \\
\hline
\end{tabular}

\section{Diagnostic Performance for TRG}

The ROC curves used to compare the diagnostic performance of T2W- $\% \triangle V$, DW- $\% \triangle V$, ceT1W- $\% \triangle V, T 2 W-\% \triangle S I R$, ceT1W- $\% \triangle S I R$, and $D W-\% \triangle V * T 2 W-\% \triangle S I R$ for assessment of predicting pathological TRG are shown in Fig. 5. Corresponding accuracy data are provided in Table 4. 
AUC, Cutoffs Value, Sensitivity, Specifcity, PPv and NPv of $\% \triangle V$ and $\% \triangle S I R$ on $T 1 W, A D C / D W I$, ceT1W between TRG.
AUC
Cutoff
Sensitivity(\%)
Specificity(\%) Accuracy(\%)
$\operatorname{PPv}(\%)$
$\mathrm{NPv}(\%)$
Value(\%)

\section{TRG 0 VS}

TRG 1

\begin{tabular}{lllllllll} 
T2W-\% $\triangle \mathrm{V}$ & $0.921(0.766,0.987)$ & 77.59 & 75.00 & 100 & 87.1 & 100 & 78.95 & .000 \\
\hline DW-\% $\triangle \mathrm{V}$ & $0.933(0.783,0.991)$ & 83.72 & 81.25 & 100 & 90.32 & 100 & 83.33 & .000 \\
ceT1W\% $\triangle \mathrm{V}$ & $0.925(0.771,0.988)$ & 77.54 & 75.00 & 100 & 87.1 & 100 & 78.95 & .000
\end{tabular}

\section{TRG 0 VS}

TRG 2

\begin{tabular}{lllllllll}
\hline T2W-\% $\triangle \mathrm{V}$ & $0.937(0.831,0.986)$ & 61.61 & 93.75 & 85.71 & 88.24 & 75 & 96.77 & .000 \\
\hline $\mathrm{DW}-\% \triangle \mathrm{V}$ & $0.946(0.845,0.990)$ & 72.02 & 93.75 & 94.29 & 94.12 & 88.24 & 97.06 & .000 \\
\hline ceT1W\% $\triangle \mathrm{V}$ & $0.939(0.835,0.987)$ & 69.43 & 87.50 & 97.14 & 94.12 & 93.33 & 94.44 & .000 \\
\hline $\begin{array}{l}\text { T2W- } \\
\% \triangle \mathrm{SIR}\end{array}$ & $0.895(0.777,0.963)$ & 29.39 & 100 & 74.29 & 82.35 & 64 & 100 & .000 \\
\hline $\begin{array}{l}\text { ceT1W- } \\
\% \triangle \mathrm{SIR}\end{array}$ & $0.746(0.605,0.858)$ & -6.42 & 100 & 54.29 & 68.63 & 50 & 100 & .005 \\
\hline $\begin{array}{l}\text { DW-\% } \triangle \mathrm{V} \text { * } \\
\text { \% } \triangle \mathrm{SIR}\end{array}$ & $0.954(0.855,0.993)$ & 0.38 & 93.75 & 97.14 & 96.08 & 93.75 & 97.14 & .000 \\
\hline
\end{tabular}

TRG 0 VS

TRG 3

\begin{tabular}{|c|c|c|c|c|c|c|c|c|}
\hline $\mathrm{T} 2 \mathrm{~W}-\% \triangle \mathrm{V}$ & $0.984(0.863,1.000)$ & 62.78 & 93.75 & 100 & 96.88 & 100 & 94.12 & .000 \\
\hline$D W-\% \triangle V$ & $0.980(0.857,1.000)$ & 68.64 & 93.75 & 100 & 96.88 & 100 & 94.12 & .000 \\
\hline ceT1W\% $\triangle \mathrm{V}$ & $0.980(0.857,1.000)$ & 60.40 & 93.75 & 100 & 96.88 & 100 & 94.12 & .000 \\
\hline $\begin{array}{l}\text { T2W- } \\
\% \triangle S I R\end{array}$ & $1.000(0.891,1.000)$ & 26.72 & 100 & 100 & 100 & 100 & 100 & .000 \\
\hline $\begin{array}{l}\text { ceT1W- } \\
\% \triangle \text { SIR }\end{array}$ & $0.859(0.691,0.956)$ & -9.23 & 100 & 81.25 & 90.63 & 84.21 & 100 & .001 \\
\hline $\begin{array}{l}\text { DW-\% } \triangle \mathrm{V} * \\
\text { T2W- } \\
\% \triangle \text { SIR }\end{array}$ & $\begin{array}{l}1.000(0.981 \\
1.000)\end{array}$ & -0.12 & 100 & 100 & 100 & 100 & 100 & .000 \\
\hline
\end{tabular}

\section{TRG 1 VS}

TRG 3

\begin{tabular}{lllllllll}
$\begin{array}{l}\text { T2W- } \\
\% \triangle \text { SIR }\end{array}$ & $0.900(0.738,0.978)$ & 24.98 & 73.33 & 100 & 87.1 & 100 & 80.00 & .000 \\
\hline $\begin{array}{l}\text { ceT1W- } \\
\% \triangle S I R\end{array}$ & $0.817(0.637,0.932)$ & -11.68 & 86.67 & 81.25 & 83.87 & 81.25 & 86.67 & .003 \\
\hline
\end{tabular}

AUC, area under the receiver operating curve; PPv, positive predictive value; NPv, negative predictive value.

There was diagnostic performance in $T 2 W-\% \triangle V$, DW- $\% \triangle V$, ceT1W-\% $\triangle \mathrm{V}$ for distinguished between TRG 0 and TRG 1 with AUC of $0.921 \sim 0.933$, sensitivity of $75.00 \sim 81.28 \%$, and specificity of $100 \%(p<0.001)$. The $T 2 W-\% \triangle V, D W-\% \triangle V$, ceT1W- $\% \triangle V$, T2W$\% \triangle S I R$, ceT1W-\% $\triangle$ SIR be used to identify between TRG 0 and TRG 2 with AUC of $0.895 \sim 0.954$, sensitivity of $87.50 \sim 100 \%$, and specificity of $54.49 \sim 97.14 \%$ ( $<<0.001$ ), as well for TRG 0 and TRG 3 with AUC of $0.859 \sim 1.000$, sensitivity of $93.75 \sim 100 \%$, and specificity of $81.25 \sim 100 \%(p<0.05)$. There was diagnostic performance in T2W- $\% \triangle S I R$, ceT1W-\% $\triangle S I R$ for distinguishing 
between TRG 1 VS TRG 3 with AUC of $0.817 \sim 0.900$, sensitivity of $73.33 \sim 86.67 \%$, and specificity of $81.25 \sim 100 \%$ ( $p<0.05)$; All parameters were not statistically different for identifying between TRG 1and TRG 2, TRG 2 and TRG 3 (Fig. 4).

\section{Comparison of diagnostic Performance for TRG}

On discriminating TRG 0 from TRG 2, the AUC of 0.937 (95\% Cl: $0.831,0.986)$ for T2W- $\% \triangle V$, 0.946 (95\% Cl: 0.845,0.990) for DW$\% \triangle \mathrm{V}, 0.939$ (95\% Cl: $0.835,0.987)$ for ceT1W-\% $\triangle \mathrm{V}$, and 0.954 (95\% Cl: $0.855,0.993)$ for DW- $\% \triangle \mathrm{V} * \mathrm{~T} 2 \mathrm{~W}-\% \triangle$ SIR with sensitivity (87.5\% 100\%), specificity (74.29\% 97.14) and accuracy (82.35\% 96.08) were signifcantly greater than the AUC of 0.746 (95\% Cl: $0.605,0.858)$ for ceT1W-\% $\triangle$ SIR, respectively $(p=0.0080 \sim 0.0154)$, Remaining AUC of ROC curves from different parameters between groups were not signifcantly different, and AUC of DW-\% $\triangle \mathrm{V} * T 2 W-\% \triangle S I R$ is slightly better than AUCs of $T 2 W-\% \triangle V, D W$ $\% \triangle V$, ceT1W-\% $\triangle \mathrm{V}, \mathrm{T} 2 \mathrm{~W}-\% \triangle \mathrm{SIR}$ for discriminating TRG 0 from TRG 2 and TRG 3.

\section{Discussion}

The focus of this study was to clarify the value of manual tumor $\% \triangle V, \% \triangle S I R$ and combined of their estimations obtained by T2W, DWI, ceT1W for predicting TRG after CRT in the patients with LARC, and results show that good diagnostic performance of $\mathrm{T} 2 \mathrm{~W}-\% \triangle \mathrm{V}, \mathrm{DW}-\% \triangle \mathrm{V}$, ceT1W-\% $\triangle \mathrm{V}, \mathrm{T} 2 \mathrm{~W}-\% \triangle \mathrm{SIR}$, ceT1W-\% $\triangle \mathrm{SIR}$, and $\mathrm{DW}-\% \triangle \mathrm{V}$ * T2W-\% $\triangle$ SIR for discriminating TRG 0 from TRG 2 and TRG 3, T2W-\% $\triangle \mathrm{V}, \mathrm{DW}-\% \triangle \mathrm{V}$, ceT1W-\% $\triangle \mathrm{V}$ for TRG 0 and TRG 1, T2W-\% $\triangle$ SIR, ceT1W-\% $\triangle$ SIR for TRG 1and TRG 3; despite there was no statistical difference in AUC of all ROC, DW- $\triangle \mathrm{V} * \mathrm{~T} 2 \mathrm{~W}-\% \triangle \mathrm{SIR}$ had highest AUC; but all measurement of $\mathrm{V}$ and SIR were not helpful for distinguishing between TRG 1 and TRG 2, TRG 2 and TRG 3. To the best of ourknowledge, no prior studies have comprehensively assessed and compared the performances of $\mathrm{V}$ change rate and SIR volume change rate on T2W, DWI, ceT1W to predict different grade response after CRT.

In present study, agreement of post-CRT MR T restaging and pathological ypT stagewas low (Kappa $=0.191)$, this is consistent with the results of some prior studies[3, 4, 8]. Tumor volume and signal intensity has been proven to be an important prognostic indicator for achange of tumors during CRT and response of after CRT. Van den et al and Doenja et al reported changes in rectal tumor morphology (fibrosis) and volume visually evaluated can already be observed during CRT[11, 16]. Volume was reported to correlate well with downstaging of rectal cancer[14, 25], and Lambregts et al reported post-CRT DWI volumetry offers the best results for the detection of patients with a CR after CRT with an area under the curve of 0.92 , sensitivity of $70 \%$, and specificity of 98\%[14]. In line with previous studies, volume has good diagnostic performance discriminating TRG 0 from TRG 1, TRG 2 and TRG 3 with AUC of $0.921 \sim 0.984$, sensitivity of $75 \sim 93.75 \%$, and specificity of $85.71 \sim 100 \%$.

Hotker el al reported tumour volumetry on post-treatment DCE-MRI and DW-MRI correlated well with histopathological percent tumour regression in the resected specimen, and was superior to post-CRT T2 tumour volumetry[8]. In present study show diagnostic performance of volume on DW was slightly greater than T2W and ceT1W, but no statistic difference between AUC of which parameters; because on DW images, viable tumor remnants are more easily recognized, as they appear hyperintense compared with the low signal intensity $(\mathrm{SI})$ of the surrounding non neoplastic tissue, which is in contrast with previous studies showed post-CRT DW MR volumetry with AUC of 0.93 provided high diagnostic performance in assessing CR and was significantly more accurate than T2-weighted MR volumetry[13].

Tumour volume may not or slightly change for poor response after CRT, but may had a fibrotic transformation that was unidentified by visual in tumor[26]. Signal intensity rate on MR parameter by quantitative as monitoring therapy response after CRT and diagnostic value for tumor characterization and differentiation[19-21, 27]. Wan et al reported T2WI signal intensity related parameters with AUC of $0.694 \sim 0.762$, sensitivity of $68.2 \% \sim 77.3 \%$, specificity of $63.6 \% \sim 77.0$ are potential predictors for pCR in LARC after CRT[12]; Value of intra-tumor heterogeneity evaluated by DW for predicting TRG to CRT in lower rectal and measurements of ADC change induced by CRT may have considerable diagnostic value for the estimation of CR, was reported[27, 28]. DCE-MRI in rectal cancer is promising mainly for prediction and assessment of response to CRT[29]. Our results are in contrast with previous studies, \% $\triangle \mathrm{SIR}$ on T2W, ceT1W is a promising diagnostic tool for CR and non-CR, TRG 1 and TRG 3 , respectively. Our $A D C-\% \triangle S I R$ results show useless, and this discrepancy in published data is probably due to the use of variety protocols, different $\mathrm{ROI}$ selection, and general factors contributing to magnetic field inhomogeneity suchas pH, hydration status, and susceptibility effects[27, 28, 30, 31]. 
In previous study, SIR and V on MR sequence was used to discriminate $\mathrm{pCR}$ or response groups from non-pCR or non response groups, and not for discrimination between TRG groups[2, 3, 8, 9, 12, 16, 18, 27, 28, 31]. In our study, SIR and $V$ change rate on T2W, DW/ADC, ceT1W first were evaluated to differentiate different response grade after CRT, and considerable results were obtained that $T 2 W-\% \triangle V, D W-\% \triangle V$, ceT1W-\% $\triangle \mathrm{V}, T 2 W-\% \triangle S I R$, ceT1W-\% $\triangle S I R$, and $D W-\% \triangle V * T 2 W-\% \triangle S I R$ has higher diagnostic performance with accuracy of $82.35 \% \sim 100 \%$ to predict TRG after pCRT compared to previous result[2, 9, 11-16, 23, 25, 27-29], except ceT1W for TRG 0 and TRG2; and among of all, despite that was no statistical difference, DW- $\% \triangle V$ * T2W-\% $\triangle$ SIR was highest for TRG with AUCs of $0.954 \sim 1.000$, sensitivity of $93.75 \% \sim 100 \%$, specificity of $97.14 \sim 100 \%$, accuracy of $96.08 \% \sim 100 \%$. $\% \triangle V$ and $\% \triangle S$ SIR not distinguished TRG 1and TRG 2, TRG 2 and TRG 3, which could be due to that volume and fibrotic transformation of tumor for grade close to grade has little change or there was a ROI selection deviations after CRT. Volume be able to distinguish TRG 0 and TRG 1, which could be due to that ROI area from TRG 0 smaller than ROI area from TRG 1 which in addition to the tumor bed, volume for ROI containa little tumor.

Despite the interesting findings, our study has some limitations. First, This was a retrospective study with asmall sample size between TRG groups, which may limit the statistical power and generate a statistical bias. Further validation may be needed by prospective study withlarge sample size to prove our hypothesis. Second, Although all data need to be collected using the same type of MRI and protocol, it was not possible to unify our data because they were obtained from multiple MR unit. Unfortunately, intraobserver and interobserver differences were not evaluated, but $\mathrm{SIR}, \triangle \mathrm{SIR}, \triangle \mathrm{V}, \% \triangle$ and $\% \triangle \mathrm{SIR}$ on $\mathrm{MR}$ sequence of preoperative after CRT for prediction TRG were calculated so as to reduce variability of imaging $[2,14,27]$. Reproducibility of relative $S I$ is relative better than $S I$ on MR sequence was reported[2, 13, 15, 19-21, 30, 31]. Hotker et al reported DCE-MRI volumetry demonstrating better inter-reader agreement[8]. Third, select of ROI and comparison of MR before and after CRT had subjectivity; to overcome these issues, we evaluated a relatively largest area of cancerous tissue[28]. Blazic et al reported the use of single-section and whole-tumor volume methods had similar accuracy in predicting CR based on post-CRT measurement ADC change and saved time compared to whole-tumor volume methods[27]

\section{Conclusion}

In conclusion, in consideration of many limitations such as $T 2 W-\% \triangle V$, DW- $\% \triangle V$, ceT1W- $\% \triangle V$, $T 2 W-\% \triangle S I R$, ceT1W- $\% \triangle S I R$, and $D W-\% \triangle V * T 2 W-\% \triangle S I R$ with good diagnostic performance showed promising results regarding TRG prediction after CRT in LARC, especially $D W-\% \triangle V * T 2 W-\% \triangle S I R$, which needs to be proven by prospective large sample cohorts. The advantage of establishing preoperative TRG as the reference standard assessment is to effectively offer management specifically tailored to patients, predict prognosis after CRT, and may include the option of non-operable management or potentially further chemoradiotherapy.

\section{Abbreviations}

LARC: locally advanced rectal cancer; CRT: chemoradiotherapy; TRG: tumor regression grade; SI: signal intensity; V: volume; MR: magnetic resonance imaging; T2W: T2-weighted; DW: diffusion-weighted; ADC: apparent diffusion coefficient; ceT1W: contrastenhanced T1-weighted; nonceT1W: non contrast-enhanced T1-weighted; $\triangle \mathrm{V}$ : difference of volume between pre-CRT and post-CRT tumor; \% $\triangle \mathrm{V}$ : V of tumor reduction rate; SIt: SI of tumor; SIm: SI of muscle; SIR: relative SI ratio of tumor/muscle; $\triangle$ SIR: changed difference SIR between pre- and post-CRT SIR; \% $\triangle$ SIR: SIR of tumor changed rate; ROC: operating characteristic curves; pCR: pathological complete remission; TME: total mesorectal excision; ROI: regions-of-interest; cT: clinical tumor stage(Topography).

\section{Declarations}

\section{Acknowledgements}

Not applicable.

\section{Author's contributions}

Xin Li designed the study; Xin Li, Zhengwu Tan, Lingling Xieand Zhenyu incollected the data; Lan Cheng collected MR scanning parameters;Xin Li,Zhengwu Tan, and Lan Zhang reviewed the imaging data; Zhengwu Tananalyzed the data, reviewed the charts, 
and interpreted the data; Xin Liand Zhengwu Tan wrote the paper; Ping Hanmodified the article.

\section{Founding}

This research was supported by the National Natural Science Foundation of China (No. 81701673) and the Natural Science Foundation of Hubei Province.

\section{Competing interests}

None of the authors have any conflicts of interest to disclose.

\section{Ethics approval and consent to participate}

This research was approved by the medical ethics committee of the Union Hospital. The requirement for informed consent was waived owing to the retrospective design of the study.

\section{References}

1. Siddiqui MRS, Bhoday J, Battersby NJ, Chand M, West NP, Abulafi A-M, Tekkis PP, Brown G: Defining response to radiotherapy in rectal cancer using magnetic resonance imaging and histopathological scales. World Journal of Gastroenterology 2016, 22(37):8414.

2. S K, K H, N S, HJ K, MJ K, WS K, JB A, JS L: T2-weighted signal intensity-selected volumetry for prediction of pathological complete response after preoperative chemoradiotherapy in locally advanced rectal cancer. European radiology 2018, 28(12):5231-5240.

3. N H, http://orcid.org I-O, C CTR, B CO, I P, MJ G, http://orcid.org I-O: MRI of Rectal Cancer: Tumor Staging, Imaging Techniques, and Management. Radiographics 2019, 39(2):367-387.

4. F S, G B, D C, A W, LST M, S B, J E, C P, R B, D T et al: Comparison between MRI and pathology in the assessment of tumour regression grade. $\mathrm{Br} J$ Cancer 2017, 117(10):1478-1485.

5. UB P, F T, L B, C G, H E, P T, P Q, D S-M, B M, R H et al: Magnetic resonance imaging-detected tumor response for locally advanced rectal cancer predicts survival outcomes: MERCURY experience. Journal of clinical oncology : official journal of the American Society of Clinical Oncology 2011, 29(28):3753-3760.

6. NJ B, B M, S Y, P T, G B: MR imaging for rectal cancer: the role in staging the primary and response to neoadjuvant therapy. Expert review of gastroenterology \& hepatology 2014, 8(6):703-719.

7. Siddiqui MR, Gormly KL, Bhoday J, Balyansikova S, Battersby NJ, Chand M, Rao S, Tekkis P, Abulafi AM, Brown G: Interobserver agreement of radiologists assessing the response of rectal cancers to preoperative chemoradiation using the MRI tumour regression grading (mrTRG). Clinical radiology 2016, 71(9):854-862.

8. AM H, L T, Y M, KM W, M G, LB S, KA G, J G-A, MJ G: Multiparametric MRI in the assessment of response of rectal cancer to neoadjuvant chemoradiotherapy: A comparison of morphological, volumetric and functional MRI parameters. European radiology 2016, 26(12):4303-4312.

9. K M, M S, A T, T N, N A, T F, H N, H O, T I, M O: Prognostic Significance of Neoadjuvant Rectal Score and Indication for Postoperative Adjuvant Therapy in Rectal Cancer Patients After Neoadjuvant Chemoradiotherapy. In vivo (Athens, Greece) 2020, 34(1):283-289.

10. RGH B-T, DMJ L, M M, S B, B B, L C-S, HM F, MJ G, S G, S H et al: Magnetic resonance imaging for clinical management of rectal cancer: Updated recommendations from the 2016 European Society of Gastrointestinal and Abdominal Radiology (ESGAR) consensus meeting. European radiology 2018, 28(4):1465-1475.

11. Lambregts DMJ, Yassien AB, Lahaye MJ, Betgen A, Maas M, Beets GL, van der Heide UA, van Triest B, Beets-Tan RGH: Monitoring early changes in rectal tumor morphology and volume during 5 weeks of preoperative chemoradiotherapy - An evaluation with sequential MRIs. Radiotherapy and Oncology 2018, 126(3):431-436.

12. LJ W, CD Z, HM Z, YK M, F Y, Y L, XM Z, CW Z: The value of MR T2WI signal intensity related parameters for predicting pathological complete response to neoadjuvant chemoradiotherapy in locally advanced rectal cancer. Zhonghua Zhong Liu 
Za Zhi 2019, 41(11):837-843.

13. L C-S, DM L, M M, T T, RT M, G L, GL B, F C-A, RG B-T: Rectal cancer: assessment of complete response to preoperative combined radiation therapy with chemotherapy-conventional MR volumetry versus diffusion-weighted MR imaging. Radiology 2011, 260(3):734-743.

14. DM L, SX R, S S, MH M, LA H, J B, M S, GL B, RA V, RG B-T: MRI and Diffusion-weighted MRI Volumetry for Identification of Complete Tumor Responders After Preoperative Chemoradiotherapy in Patients With Rectal Cancer. Ann Surg 2015, 262(6):1034-1039.

15. M P, R F, O C, M S, A A, P D, B P, F T, A P: MRI for Assessing Response to Neoadjuvant Therapy in Locally Advanced Rectal Cancer Using DCE-MR and DW-MR Data Sets: A Preliminary Report. BioMed research international 2015, 2015:514740.

16. R VdB, http://orcid.org I-O, JP K, http://orcid.org I-O, B E, M P, B vA, B R, O R, M DR et al: Tumor volume regression during preoperative chemoradiotherapy for rectal cancer. Acta Oncol 2018, 57(6):723-727.

17. M I, EM M, O R, ME P: Combined T2w volumetry, DW-MRI and DCE-MRI for response assessment after. Acta Oncol 2015, 54(10):1729-1736.

18. Cao W, Li B, Gong J, Hu M, Li W, Pan X, Zhou Z: Diffusion-weighted magnetic resonance imaging of mucin pools in locally advanced rectal mucinous adenocarcinoma predicts tumor response to neoadjuvant therapy. European journal of radiology $2020,125: 108890$.

19. H N, M L, VR M, T P, M B: Diagnostic Value of Diffusion-Weighted MRI for Tumor Characterization, Differentiation and Monitoring in Pediatric Patients with Neuroblastic Tumors. RoFo : Fortschritte auf dem Gebiete der Rontgenstrahlen und der Nuklearmedizin 2017, 189(7):640-650.

20. D H, J L, J B, M R, M O, M M, A S, R G, R S, SO S et al: Image Quality Assessment of 2D versus 3D T2WI and Evaluation of Ultra-high b-Value (b=2,000 mm/s2) DWI for Response Assessment in Rectal Cancer. Anticancer Res 2018, 38(2):969-978.

21. TH K, Y H, JJ S, YE C, JH L, WH C: Signal intensity ratio on magnetic resonance imaging as a prognostic factor in patients with cervical compressive myelopathy. Medicine 2016, 95(39):e4649.

22. SB E, CC C: The American Joint Committee on Cancer: the 7th edition of the AJCC cancer staging manual and the future of TNM. Annals of surgical oncology 2010, 17(6):1471-1474.

23. R R, D G, JM H, D T, A W, HE M, DP OD, M M, D F, K S: Pathological response following long-course neoadjuvant chemoradiotherapy for locally advanced rectal cancer. Histopathology 2005, 47(2):141-146.

24. ER D, DM D, DL C-P: Comparing the areas under two or more correlated receiver operating characteristic curves: a nonparametric approach. Biometrics 1988, 44(3):837-845.

25. DMJ L, TN B, RGH B-T: Response evaluation after neoadjuvant treatment for rectal cancer using modern MR imaging: a pictorial review. Insights Imaging 2019, 10(1):019-0706.

26. M M, RAP D, R B-T: Rectal Cancer: Assessing Response to Neoadjuvant Therapy. Magn Reson Imaging Clin N Am 2020, 28(1):117-126.

27. IM B, GB L, MM G: Quantitative Assessment of Rectal Cancer Response to Neoadjuvant Combined Chemotherapy and Radiation Therapy: Comparison of Three Methods of Positioning Region of Interest for ADC Measurements at Diffusionweighted MR Imaging. Radiology 2017, 282(2):418-428.

28. M K, M N, Y K, Y M, T A, M K, E K, M G, K Y, E O: Value of intra-tumor heterogeneity evaluated by diffusion-weighted MRI for predicting pathological stages and therapeutic responses to chemoradiotherapy in lower rectal cancer. Journal of Cancer 2020, 11(1):168-176.

29. RAP D, RGH B-T, DMJ L, GL B, M M: Value of DCE-MRI for staging and response evaluation in rectal cancer: A systematic review. European journal of radiology 2017, 95:155-168.

30. AB R, M O, JS B, BE N, B T: Diffusion-weighted imaging of the abdomen at 3.0 Tesla: image quality and Apparent Diffusion Coefficient Reproducibility Compared With 1.5 Tesla. J Magn Reson Imaging 2011, 33(1):128-135.

31. G B, S B, A C, A C, M E, M G, LN M, L N, R S, R T et al: Quality assurance multicenter comparison of different MR scanners for Quantitative Diffusion-Weighted Imaging. J Magn Reson Imaging 2016, 43(1):213-219.

Page 12/16 


\section{Figures}

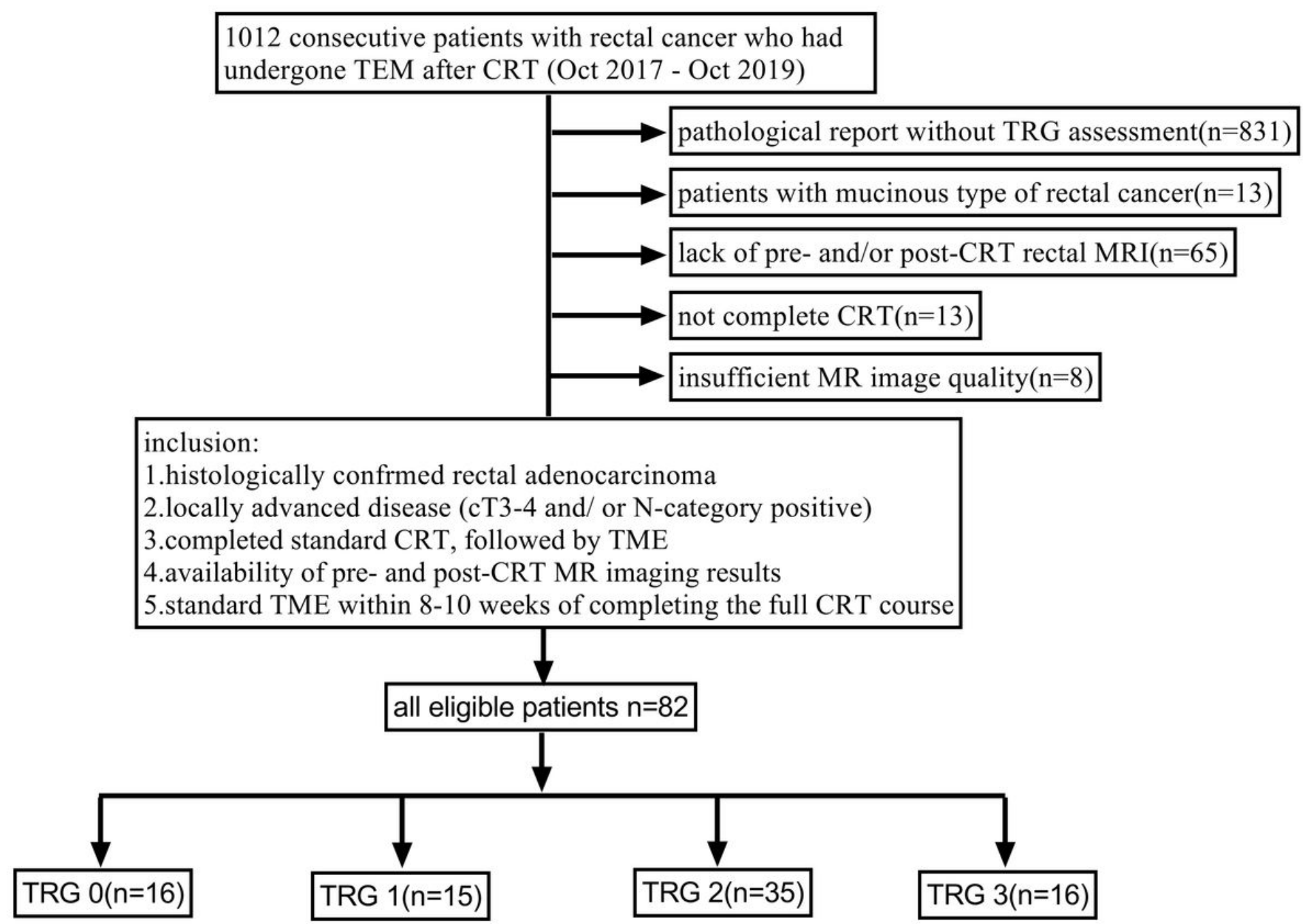

Figure 1

Flow diagram of the studypopulation 


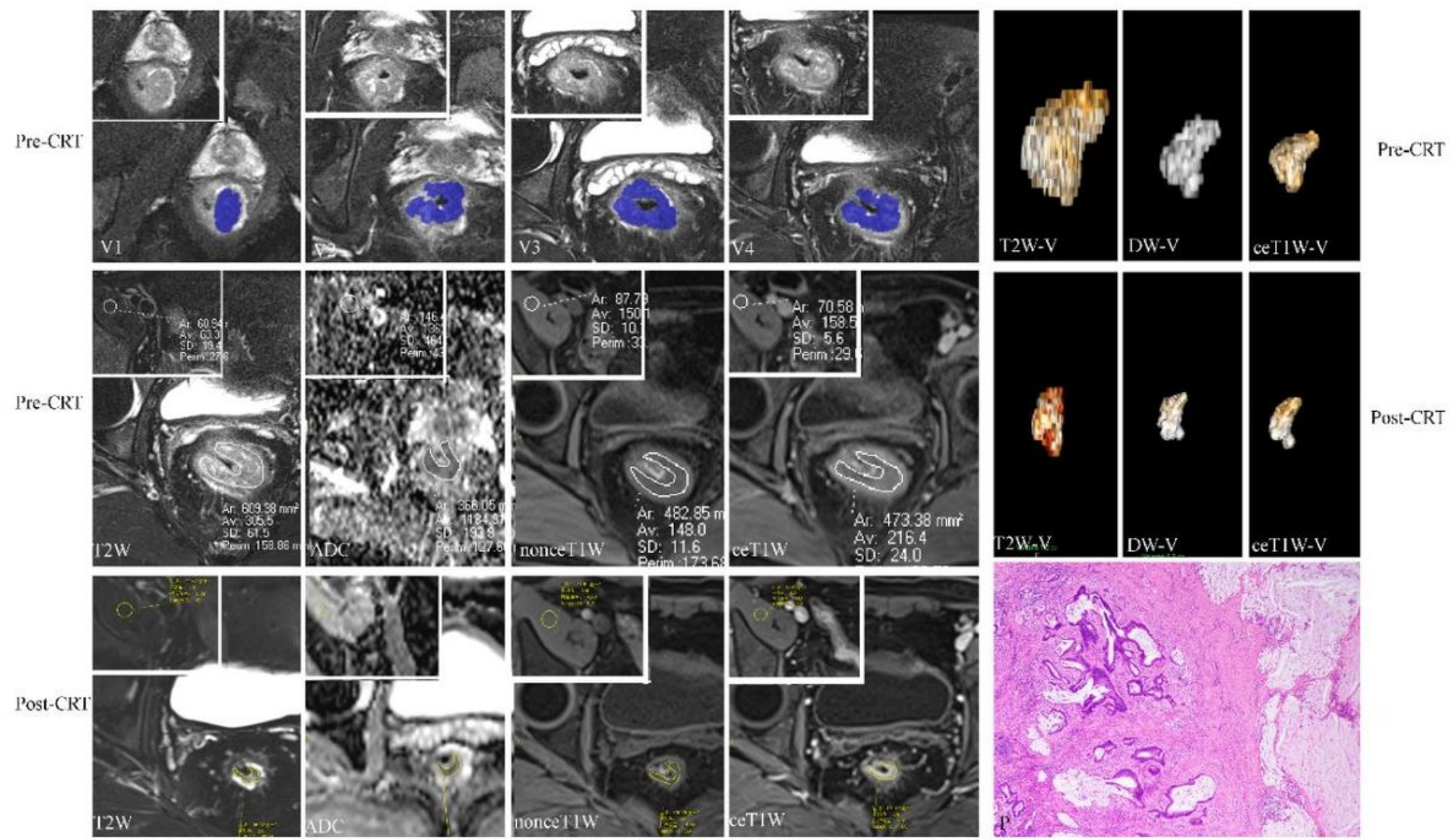

Figure 2

Examples of the pre-CRT and corresponding post-CRT of quantitative volumetric and signal intensity measurements performed in a 63-year-old male patient with advanced rectal cancer who first was histologically confirmed moderately differentiated adenocarcinoma, diagnosed T4 based on MR and post-CRT diagnosed T2. Whole-tumor volume was calculated by multiplying each crosssectional area by section thickness (V1-V4), and signal intensity of tumor was measured by relatively largest ROI of cancerous tissue obtained on single same sections of axial T2W, ADC, nonceT1W, ceTW, as well as for measurement of signal intensity of muscle (The inset in the upper leftcorner). Post-CRT measurements of signal intensity were performed with comparison to pre-CRT MR images to ensure ROls were placed within same axial level of the location of the primary tumor. Interval between pre- and post-CR MR was 115 day and interval between post-CRT MR and surgery was 4 day. Compared pre-CRT MR and post-CRT MR, tumor had decreased $71.08 \%$ in $T 2 W-\% \triangle V, 64.89 \%$ in $D W-\% \triangle V, 68.72 \%$ in ceT1W\% $\triangle \mathrm{V},-27.81 \%$ in $T 2 W-$ $\% \triangle S I R,-65.24 \%$ in ceT1W-\% $\triangle$ SIR, respectively, and final histologically confirmed ypT2 and TRG $2(P)$.

Pre-CRT
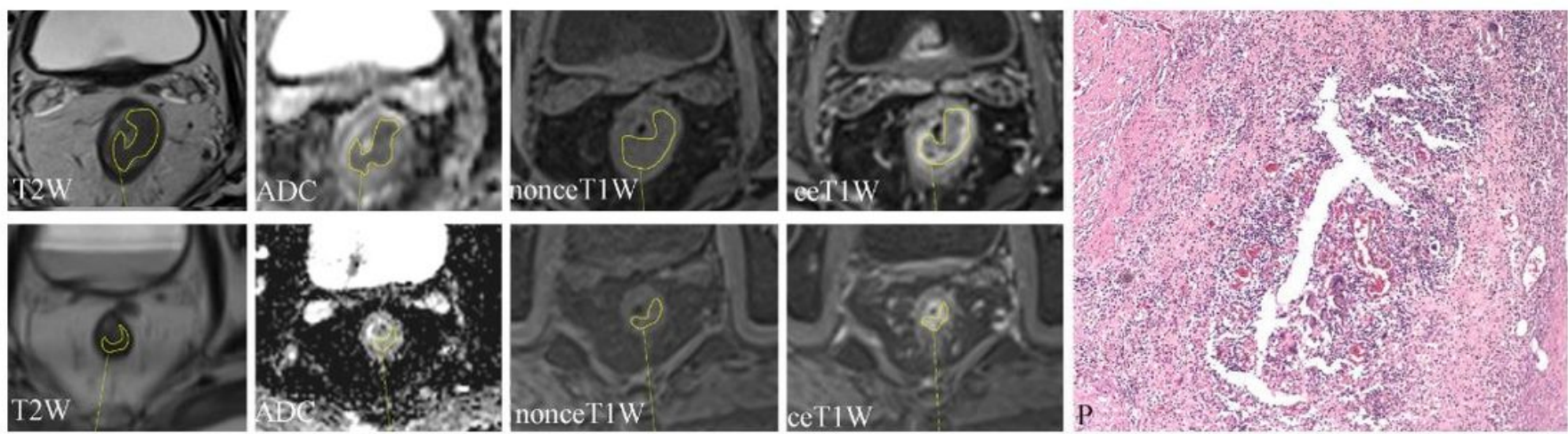

Figure 3 
Examples of the pre-CRT and corresponding post-CRT of quantitative signal intensity measurements performed in a 52-year-old male patient with advanced rectal cancer who first was histologically confirmed moderately differentiated adenocarcinoma, diagnosed T4 based on MR and post-CRT diagnosed T2. The patient has a well-defined, almost circular tumor mass (Pre-CRT). Post-CRT, tumor signal-intensity zones were not identifed on T2W, ADC, nonceT1W, ceT1W images, and then the ROIs were positioned at the location of the tumor bed before CRT, comparison to pre-CRT MR images. Interval between pre- and post-CR MR was 91 day and interval between post-CRT MR and surgery was 9 day. Comparedpre-CRT MR and post-CRT MR, tumor had decreased $97.77 \%$ in T2W-\% $\triangle \mathrm{V}, 94.29 \%$ in DW-\% $\triangle \mathrm{V}, 81.37 \%$ in ceT1W\% $\triangle \mathrm{V}, 46.21 \%$ in $\mathrm{T} 2 \mathrm{~W}-\% \triangle \mathrm{SIR},-5.76 \%$ in ceT1W- $\% \triangle S I R$, respectively, and final histologically confirmed ypT0 and TRG 0 (P).

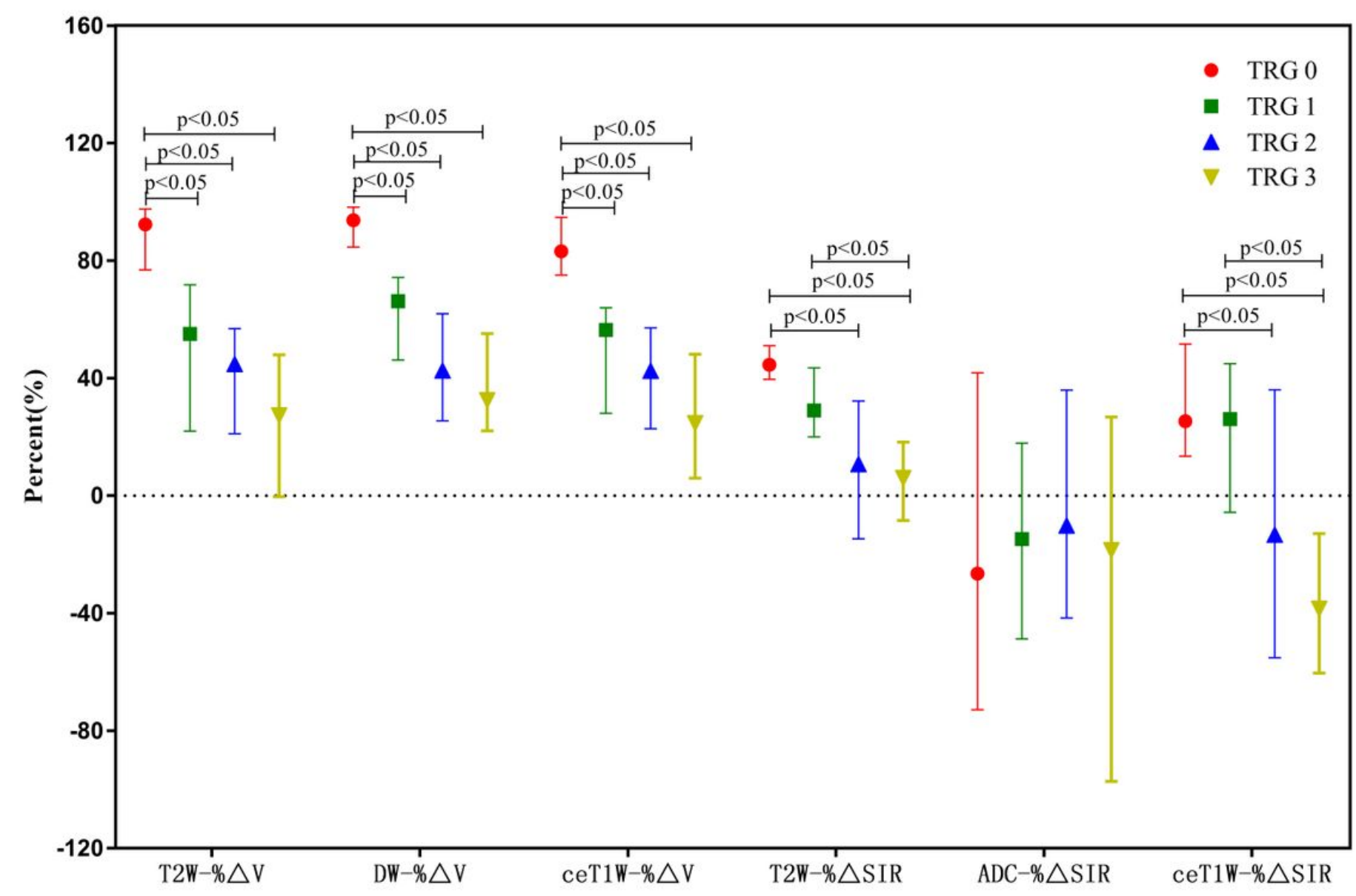

Figure 4

Box and whisker plots represent distribution of $\% \triangle V$ and $\% \triangle S I R$ in different TRG groups. Middle line in each box represents the median value of $\% \triangle V$ and $\% \triangle S I R$. Lower and upper boundaries of the boxes represent the first and third quartiles (25th and 75th percentiles), respectively. 
TRG 0 VS. TRG 1

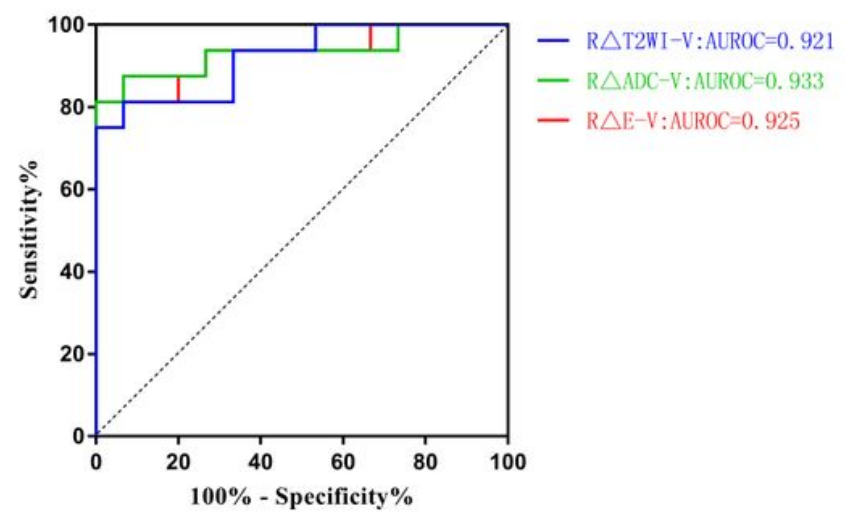

TRG 0 VS. TRG 3

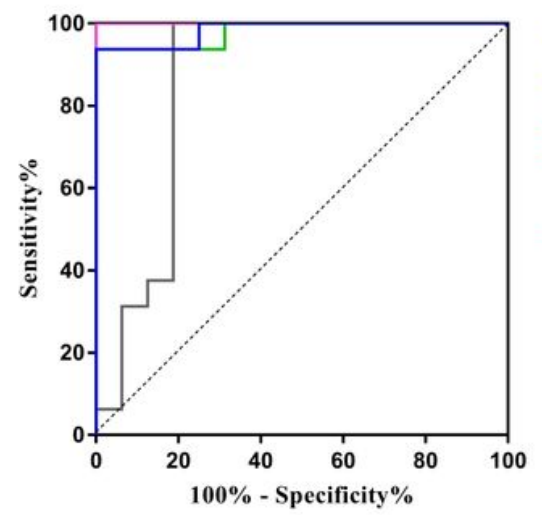

- R $\triangle T 2 W I-V: A U R O C=0.984$

- R $\triangle A D C-V: A U R O C=0,980$

- R $\triangle E-V: A U R O C=0.980$

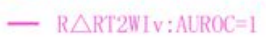

- R $\triangle$ REDv:AUROC $=0.859$

- R $\triangle A D C-V * R \triangle R T 2 W I v: A U R O C=1$
TRG 0 VS. TRG 2

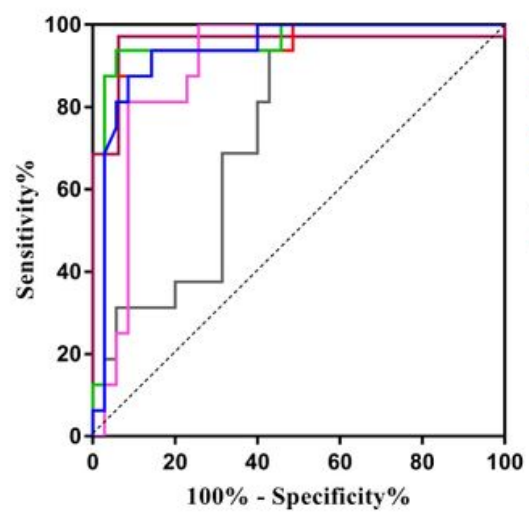

- R $\triangle T 2 W I-V: A U R O C=0.937$

- R $\triangle A D C-V: A U R O C=0.946$

- R $\triangle E-V: A U R O C=0.939$

- R $\triangle R T 2 W I v: A L R O C=0.895$

- R $\triangle$ REDv:AUROC $=0.746$

- R $\triangle A D C-V * R \triangle R T 2 W I v: A U R O C=0.954$

\section{Figure 5}

Receiver operating characteristic ccurves using $\% \triangle V$ and $\% \triangle S I R$ on $T 1 W, A D C / D W I$, ceT1W between pairwise comparison.

\section{Supplementary Files}

This is a list of supplementary files associated with this preprint. Click to download.

- AppendixA2.docx

- AppendixA2.docx

- AppendixA1.docx

- AppendixA1.docx 